\title{
Use of the Americans in Motion-Healthy Intervention (AIM-HI) to Create a Culture of Fitness in Family Practice
}

\author{
Jamie Erskine, PhD, RD, Angela Lanigan, MPA, Caroline B. Emsermann, MS, \\ Brian K. Manning, MPH, Elizabeth W. Staton, MSTC, and Wilson D. Pace, MD
}

Background: Americans in Motion-Healthy Interventions (AIM-HI) is an initiative designed to assist family physicians with positioning fitness (physical activity, nutrition, and emotional well-being) as the treatment of choice for prevention and management of chronic disease. We investigated whether the concept of a culture of fitness would benefit office personnel and carry over to patient care.

Metbods: This randomized, controlled trial provided an intervention based on the AIM-HI curriculum to 12 enhanced offices with support for office activities, while 12 traditional offices received only AIM-HI tools with encouragement for use with patients. Before intervention, at 4 months, and at 14 months, we measured the practice personnel's dietary behavior (PrimeScreen), physical activity (International Physical Activity Questionnaire), self-determined (intrinsic) motivation (Treatment Self-Regulation Questionnaire [TSRQ]), perceived ability to carry out health behaviors (Perceived Competence Scale), and readiness to improve and/or maintain health behaviors (Stages of Change).

Results: From 24 practices we enrolled 470 subjects; 21 practices completed the study, and data from 341 patients were analyzed. Differential change from baseline between the enhanced and traditional offices was not evident for behavior changes. An overall decrease from baseline in self-reported total physical activity measured as metabolic equivalent-minutes for all surveyed groups occurred over the study time period (4-month $\beta=-11.97 ; 14$-month $\beta=-9.01 ; P=.003$ ). A statistically significant increase occurred at 4 months among participants from the enhanced practices for the TSRQ outcomes of Healthy Eating (baseline, $3.00 \pm 0.12 ; 4$ months, $3.26 \pm 0.13 ; P=.013$ ). Among clinicians, TSRQ Healthy Eating scores increased from $3.19 \pm 0.13$ at baseline to $3.52 \pm 0.14$ at 4 months $(P=$ $.005)$. However, increases in TSRQ Eating scores were not sustained by 14 months. Stages of Change scores decreased from baseline to 4 months in enhanced group offices. There was also a decrease in Stages of Change scores among staff from baseline to 14 months.

Conclusions: Primary care clinicians and office staff are resistant to health behavior change. External motivation did not seem to help them change. The effect of this intervention on patient care is not yet known. (J Am Board Fam Med 2012;25:694-700.)

Keywords: Family Medicine Research, Lifestyle Modification, Practice-based Research, Practice-based Research Networks, Workplace Wellness

Americans in Motion-Healthy Interventions (AIM$\mathrm{HI}$ ) is an initiative of the American Academy of Family Physicians that was designed to assist family

This article was externally peer reviewed.

Submitted 28 February 2011; revised 16 March 2012; accepted 23 May 2012.

From the Nutrition and Dietetics Program, University of Northern Colorado, Greenley, (JE); the American Academy of Family Physicians (AAFP) National Research Network, Leawood, KS (AL, BKM, EWS, WDP); and the Department of Family Medicine, University of Colorado School of Medicine, Aurora, (CBE). physicians with positioning fitness (physical activity, nutrition, and emotional well-being) as the treatment of choice for prevention and management of many chronic conditions. ${ }^{1}$ The tools and

Funding: Funding was provided through a grant to the American Academy of Family Physicians from the Pepsico Foundation via the AAFP Foundation.

Conflict of interest: none declared.

Corresponding author: Wilson Pace, MD, University of Colorado, Academic Office One, Rm 3405, Campus Box F496, Denver, CO 80045(E-mail: wilson.pace@ucdenver.edu). 
resources associated with this initiative, based in part on the 5 As model, ${ }^{2}$ encourage clinicians to create an office culture that promotes healthy behaviors and relationships with patients that promote healthy behavior change. This goal coincides with Healthy People 2020 objectives of increasing counseling about physical activity and nutrition during office visits. ${ }^{3}$

AIM-HI encourages multistep implementation. An important first step of the process is to create awareness among physicians and office staff of their own personal nutrition, physical activity, and emotional well-being choices before working with patients. At first glance, this step may seem to be a typical workplace wellness program, but the goal is to enhance self-awareness of personal lifestyle choices so that clinicians develop a more patientcentered approach when working on fitness goals with patients. Greater self-awareness by clinicians also may increase clinicians' willingness to have fitness discussions with patients, ${ }^{4-6}$ yet few studies have examined onsite interventions for promoting healthy behaviors among clinicians. ${ }^{7-9}$

In addition to creating awareness, clinicians are encouraged to make simple behavior changes in their own lives. Potential benefits include (1) clinicians becoming positive role models for their patients, (2) patients being more likely to perceive their clinicians as credible when clinicians discuss health behaviors, and (3) clinicians being more likely to have fitness-related discussions with their patients if the clinicians themselves practice healthy behaviors. ${ }^{8,10}$

Practices are interested in creating a healthier office culture for patients, clinicians, and staff. ${ }^{9}$ To date, the AIM-HI program has been well received by a number of family practices. However, there has not been a thorough assessment of the program's impact on participants' health behavior and related outcomes. The goal of this study was to assess the effects of a health promotion program (AIM-HI) on clinicians' and staff's health behaviors and attitudes. We hypothesized that clinicians and staff from enhanced practices-those that were encouraged and supported to become familiar with health promotion tools, use the tools to make their own personal lifestyle changes, and create a fitness culture within their own practices-would show greater change in the self-reported outcomes than those from traditional offices.

\section{Methods \\ Research Design}

This study was a part of a randomized, controlled trial involving clinicians and staff at family medicine practices. Practices receiving the intervention were designated as "enhanced offices" and were compared with usual practice or "traditional offices." We also compared health behaviors and attitudes of clinicians and staff as a whole. This study was reviewed and approved by the American Academy of Family Physicians Institutional Review Board. Staff and clinicians were required to sign informed consent before participating.

\section{Setting and Participants}

Twenty-four practices were recruited from members of 3 practice-based research networks, including the American Academy of Family Physicians National Research Network, LA Net, and the Southeast Clinicians Research Network. An inclusion criterion was that at least $75 \%$ of the total staff per practice agreed to participate. To achieve representation across regions of the United States, 24 practice offices located in 16 states were asked to participate. The practices were randomized to include stratification with respect to size (small was defined as $\leq 3$ clinicians and large as $\geq 4$ clinicians) and the percentage of minority patients, with at least $35 \%$ minority as the dividing point. Identities of the staff and clinician respondents were kept anonymous to the researchers, but the study coordinator at each site maintained a list matching individuals to survey identification numbers for purposes of comparing data across time.

\section{Intervention}

Practices $(\mathrm{N}=24)$ were randomized to either the "enhanced office" group or the "traditional office" group. The groups were not blinded to their assignment. A lead physician and study coordinator from each practice were introduced to the AIM-HI approach and materials (described later) at an inperson training session with other practices, research staff, and study investigators. The 12 practices randomized to the enhanced office group attended the training session 3 months before implementing AIM-HI with patients.

During the training, research staff helped "enhanced offices" strategize and plan how they would implement office activities that encouraged staff 
and clinicians to pursue healthy lifestyle goals. Research staff supported practice efforts throughout the course of the study through biweekly phone calls and emails to the practice coordinators. In this way, staff and clinicians in enhanced practices were encouraged and supported to become familiar with health promotion tools, use the tools to make their own personal lifestyle changes, and create a fitness culture within their own practices.

Practices in the enhanced group established group activities that varied by office to promote fitness and health. A variety of activities were launched by practices, with varying frequency and duration. Examples of such activities included monthly healthy snack days, weekly "healthy" potlucks for employees, a one-time staff $5 \mathrm{~K}$ run, staff fitness buddies, group walks, purchasing a treadmill for the break room, and a one-time book exchange. These practices also received AIM-HI t-shirts, walking socks, and pedometers for all site personnel.

The 12 practices in the traditional group attended a training session just before the implementing AIM-HI with patients. The lead physicians and study coordinators were trained in the use of the AIM-HI tools and encouraged to use them only with patients. Although they were not discouraged from participating in office wellness activities with staff and clinicians, no training time was devoted to planning office activities. Research staff members did not assist in carrying out office activities or provide additional materials as they did for offices in the enhanced group.

Health education tools developed for this project were modeled on the 5 As and Transtheoretical models and were designed to support incremental behavior changes. ${ }^{11,12}$ The tools included a fitness inventory, fitness prescription, fitness promotion posters, and a food and activity journal. ${ }^{1}$ The tools were developed by the AIM-HI educational team, which included individuals with expertise in family medicine, health education, technical communication, physical activity, and weight loss. The fitness inventory assessed physical activity, emotional health, and dietary behavior and readiness to change and/or maintain health behaviors. The fitness inventory includes questions from validated measures such as the International Physical Activity Questionnaire-Short Form (IPAQ), PrimeScreen, and the Stages of Change assessment. ${ }^{1,12-15}$ The fitness prescription was modeled on behavior change prescriptions for smoking cessation and resembles a medicine/ treatment prescription. In addition, the AIM-HI approach encouraged the use of motivational interviewing principles to help establish realistic and achievable goals. ${ }^{16}$ All items were adapted for primary care use by the AIM-HI educational team.

\section{Data Collection}

A Clinician Health Survey was administered 3 times over the course of the study: at baseline (before the enhanced office training), 4 months after training, and 14 months after training (Figure 1). The Clinician Health Survey was an assemblage of several validated surveys: the PrimeScreen, ${ }^{14}$ IPAQ, ${ }^{13}$ Treatment Self-Regulation Questionnaire (TSRQ), ${ }^{17,18}$ Perceived Competence Scales (PCS), ${ }^{19,20}$ and Stages of Change-Short Form. ${ }^{15,21}$

The PrimeScreen assesses dietary intake by items in food groups ranked according to frequency of intake. The IPAQ assesses physical activity and included a self-assessment of number of days per week, estimated minutes per day, and reported intensity of physical activity. TSRQ survey items assess the degree to which one's motivation for a health behavior is relatively autonomous or self-determined. PCS survey items assess feelings of competence about engaging in a health behavior. The Stages of Change survey

Figure 1. Timeline of clinician/staff activities.

\begin{tabular}{|c|c|c|c|c|c|c|c|c|c|c|}
\hline Activity & Aug 07 & Oct 07 & Dec 07 & Feb 08 & Apr 08 & Jun 08 & Aug 08 & Oct 08 & Dec 08 & Feb 09 \\
\hline Enhanced Office Training & $\mathrm{X}$ & & & & & & & & & \\
\hline & & & & & & & & & & \\
\hline Traditional Office Training & & $\mathrm{X}$ & & & & & & & & \\
\hline Enhanced Office Activities & $\mathrm{X}$ & $\mathrm{X}$ & $\mathrm{X}$ & $\mathrm{X}$ & $\mathrm{X}$ & $\mathrm{X}$ & $\mathrm{X}$ & $\mathrm{X}$ & $\mathrm{X}$ & $\mathrm{X}$ \\
\hline Health Survey Administration & $\mathrm{X}$ & & $\mathrm{X}$ & & & & & $\mathrm{X}$ & & \\
\hline Research Staff Support of Activities & $\mathrm{X}$ & $\mathrm{X}$ & $\mathrm{X}$ & $\mathrm{X}$ & $\mathrm{X}$ & $\mathrm{X}$ & & & & \\
\hline
\end{tabular}


items assess participants' readiness to improve a health behavior, maintain a health behavior, or both.

\section{Statistical Analysis}

A total of 6 composite scores were used in the analysis to measure health behavior and attitudes and changes over time. The composite dietary PrimeScreen and IPAQ as total minutes of metabolic equivalents (MET-minutes) were used to measure health behavior changes. The composite TSRQ Healthy Eating, TSRQ Physical Activity, Stages of Change, and PCS score was used to measure attitude changes.

Linear mixed-effects repeated measures models, adjusting for practices as a random effect, were performed for each of the composite scores. Two sets of models were run, testing for differential change over time from baseline between either the randomized practice groups (traditional office versus enhanced office) or the clinician versus office staff groups. The clinician group included physicians, nurses, and other practitioners whereas all other participants (eg, receptionists, billing staff, practice managers) were categorized as office staff. Each model adjusted for time point (baseline, 4 months, 14 months); traditional versus enhanced group; employee type (clinician vs staff); and time point by intervention interaction. Before the analyses, mixed models adjusting only for survey determined a nonlinear relationship between time and all outcomes. Therefore, time was run as a categorical predictor. To determine change in scores over time by each employee and practice group, one degree of freedom hypotheses tests were made a priori. Hypotheses tested for each group level whether scores increased from baseline to 4 months and 14 months. Because the distribution of the IPAQ measure was non-normal, a square-root transformation was made.

Statistically significant associations were determined at the $\alpha=0.05$ level. For one degree of freedom hypothesis, because multiple tests were being performed, the level of significance was determined at $\alpha=0.01$. All analyses were performed in SAS version 9.2 (SAS, Inc., Cary, NC). ${ }^{22}$

\section{Results}

Of the 24 original practice sites, 21 completed the study. Three practice sites dropped out because of practice coordinator attrition. Two practice sites in the same organization were combined as one for analyses because they shared physicians and staff extensively. A total of 470 subjects were enrolled from 24 different practices. Of these, data for 129 subjects were not included in the analysis because baseline data was missing. Thus, the analysis reflects data from 341 participants.

\section{General Results}

A differential change from baseline at 4 months or 14 months was not observed between the enhanced and the traditional offices for any of the outcomes. In addition, there was not a differential change between clinicians and office staff. An overall decrease from baseline in self-reported total physical activity, measured as MET-minutes, for all surveyed groups occurred over the study time period (enhanced vs traditional: 4-month $\beta=-7.27,14-$ month $\beta=-2.81 ; P=.051$; clinician vs staff: 4-month $\beta=-11.97,14$-month $\beta=-9.01 ; P=$ .003). In addition, an overall decrease from baseline in self-reported Stages of Change scores occurred among clinicians and staff (4-month $\beta=-0.21$, 14-month $\beta=-0.26 ; P=.014)$.

\section{One Degree of Freedom Hypotheses}

One degree of freedom hypotheses determined that in the enhanced group of practices, IPAQ scores decreased at both time points from baseline among personnel from enhanced practices (1601 at baseline, 1087 at 4 months $[P=.002]$, and 988 at 14 months $[P<.001]$ ). TSRQ (selfdetermined motivation) Healthy Eating scores increased from baseline (3.00) to 4 months (3.26; $P=.013)$ in enhanced practices only. Stages of Change scores decreased at 4 months in enhanced practices only (2.11 at baseline to 1.92 at 4 months; $P=.003$ ). Other outcomes did not change significantly (see Table 1).

When comparing clinicians and other staff, average self-reported IPAQ scores decreased at both time points from baseline among staff members but not clinicians (staff: 1602 at baseline, 787 at 4 months $[P=.001]$, and 962 at 14 months $[P=$ .012]). TSRQ (self-determined motivation) Healthy Eating scores increased for clinicians from baseline (3.19) to 4 months $(3.52 ; P=.005)$. Stages of Change scores decreased at 14 months from baseline among staff members (2.26 at baseline to 
Table 1. One Degree of Freedom Hypotheses: Enhanced versus Traditional Offices

\begin{tabular}{|c|c|c|c|c|c|}
\hline Survey Outcome by Intervention & Baseline & 4 Months & $P$ & 14 Months & $P$ \\
\hline \multicolumn{6}{|l|}{$\mathrm{IPAQ}^{*}$} \\
\hline Traditional & $1365 \pm 6.77$ & $880 \pm 6.69$ & .017 & $1165 \pm 7.22$ & .364 \\
\hline Enhanced & $1601 \pm 4.64$ & $1087 \pm 3.99$ & $.002^{\dagger}$ & $988 \pm 4.58$ & $<.001^{\dagger}$ \\
\hline \multicolumn{6}{|l|}{ PrimeScreen } \\
\hline Traditional & $-0.01 \pm 0.05$ & $-0.01 \pm 0.06$ & .927 & $-0.01 \pm 0.06$ & .849 \\
\hline Enhanced & $0.02 \pm 0.05$ & $0.08 \pm 0.05$ & .018 & $0.02 \pm 0.05$ & .969 \\
\hline \multicolumn{6}{|l|}{ TSRQ: Healthy Eating } \\
\hline Traditional & $3.27 \pm 0.15$ & $3.49 \pm 0.17$ & .159 & $3.30 \pm 0.18$ & .889 \\
\hline Enhanced & $3.00 \pm 0.12$ & $3.26 \pm 0.13$ & $.013^{\dagger}$ & $3.09 \pm 0.14$ & .407 \\
\hline \multicolumn{6}{|l|}{ TSRQ: Physical Activity } \\
\hline Traditional & $3.44 \pm 0.16$ & $3.56 \pm 0.18$ & .453 & $3.41 \pm 0.19$ & .878 \\
\hline Enhanced & $3.19 \pm 0.12$ & $3.43 \pm 0.14$ & .040 & $3.25 \pm 0.15$ & .662 \\
\hline \multicolumn{6}{|l|}{ Stages of Change } \\
\hline Traditional & $1.98 \pm 0.10$ & $2.00 \pm 0.12$ & .830 & $1.86 \pm 0.12$ & .190 \\
\hline Enhanced & $2.11 \pm 0.09$ & $1.92 \pm 0.10$ & $.003^{\dagger}$ & $2.04 \pm 0.10$ & .365 \\
\hline \multicolumn{6}{|l|}{ PCS } \\
\hline Traditional & $4.04 \pm 0.09$ & $3.99 \pm 0.11$ & .688 & $4.00 \pm 0.12$ & .764 \\
\hline Enhanced & $3.81 \pm 0.07$ & $3.88 \pm 0.08$ & .381 & $3.75 \pm 0.09$ & .540 \\
\hline
\end{tabular}

Values provided as mean $\pm \mathrm{SE}$.

*Transformed to original units.

${ }^{\dagger}$ Significant at $P \leq 0.01$.

IPAQ, International Physical Activity Questionnaire-Short Form; PCS, Perceived Competence Scales; TSRQ, Treatment SelfRegulation Questionnaire.

2.01 at 14 months; $P=.010)$. Other outcomes did not change significantly (see Table 2).

\section{Discussion}

We found little effect of the intervention on improving health behaviors and attitudes over the follow-up period. The intervention was most successful in improving internal motivation to eat a healthy diet. In the enhanced practices and among clinicians, TSRQ Healthy Eating scores increased from baseline to 4 months. Internal motivation is associated with positive health behaviors and health outcomes, ${ }^{23}$ but in this study dietary behaviors did not improve. Improvement in TSRQ Healthy Eating scores was not sustained at 14 months. There were no other significant improvements when comparing enhanced with traditional practices.

IPAQ (physical activity) scores decreased over time among personnel from enhanced practices and the staff group as a whole. IPAQ scores may have dropped between baseline and month 4 because of seasonal variation in physical activity. The baseline survey was distributed in late summer, whereas the month 4 survey was handed out in winter, during which fewer daylight hours and cold weather make it more difficult for people to participate in physical activity. Stages of Change scores also decreased at 4 months in enhanced offices. This suggests that personnel from enhanced offices were less ready to make changes in dietary behaviors and physical activity at 4 months. In the clinician group, Stages of Change scores decreased significantly from baseline to 14 months.

It is well established that an intensive intervention with frequent, long-term interaction is more successful than shorter or one-time interventions. ${ }^{24-27}$ Biweekly contact with the enhanced offices after the initial in-person practice training would seem to be adequate for busy family medicine practices; however, the follow-up was by phone and e-mail, and each practice was permitted to implement activities fostering a fitness culture on an office-by-office basis. For these analyses we did not include a measure of the fidelity and intensity of AIM-HI staff wellness activities that were implemented. Further analyses are warranted to better understand how and which wellness programs make a difference. We can suggest that, overall, follow-up needs to be further tailored in 
Table 2. One Degree of Freedom Hypotheses: Clinicians versus Staff

\begin{tabular}{|c|c|c|c|c|c|}
\hline Survey Outcome by Group & Baseline (mean $\pm \mathrm{SE}$ ) & 4 Month (mean $\pm \mathrm{SE})$ & $P$ & 14 Month (mean $\pm \mathrm{SE})$ & $P$ \\
\hline \multicolumn{6}{|l|}{$\mathrm{IPAQ}^{*}$} \\
\hline Staff & $1602 \pm 9.05$ & $787 \pm 6.64$ & $.001^{\dagger}$ & $962 \pm 7.58$ & $.012^{\dagger}$ \\
\hline Clinician & $1473 \pm 4.02$ & $1157 \pm 3.81$ & .045 & $1130 \pm 4.34$ & .043 \\
\hline \multicolumn{6}{|l|}{ PrimeScreen } \\
\hline Staff & $-0.12 \pm 0.05$ & $-0.05 \pm 0.05$ & .069 & $-0.08 \pm 0.05$ & .366 \\
\hline Clinician & $0.08 \pm 0.04$ & $0.10 \pm 0.04$ & .469 & $0.06 \pm 0.04$ & .568 \\
\hline \multicolumn{6}{|l|}{ TSRQ: Health Eating } \\
\hline Staff & $3.11 \pm 0.16$ & $3.26 \pm 0.18$ & .349 & $3.13 \pm 0.19$ & .916 \\
\hline Clinician & $3.19 \pm 0.13$ & $3.52 \pm 0.14$ & $.005^{\dagger}$ & $3.31 \pm 0.15$ & .354 \\
\hline \multicolumn{6}{|l|}{ TSRQ: Physical Activity } \\
\hline Staff & $3.35 \pm 0.17$ & $3.36 \pm 0.19$ & .938 & $3.36 \pm 0.19$ & .939 \\
\hline Clinician & $3.40 \pm 0.13$ & $3.67 \pm 0.14$ & .022 & $3.43 \pm 0.15$ & .826 \\
\hline \multicolumn{6}{|l|}{ Stages of Change } \\
\hline Staff & $2.26 \pm 0.09$ & $2.05 \pm 0.11$ & .021 & $2.01 \pm 0.11$ & $.010^{\dagger}$ \\
\hline Clinician & $1.94 \pm 0.08$ & $1.87 \pm 0.09$ & .287 & $1.93 \pm 0.09$ & .791 \\
\hline \multicolumn{6}{|l|}{ PCS } \\
\hline Staff & $3.75 \pm 0.10$ & $3.85 \pm 0.11$ & .423 & $3.75 \pm 0.12$ & .973 \\
\hline Clinician & $4.00 \pm 0.08$ & $4.00 \pm 0.09$ & .926 & $3.94 \pm 0.09$ & .568 \\
\hline
\end{tabular}

*Transformed to original units.

${ }^{\dagger}$ Significant at $P \leq 0.01$.

IPAQ, International Physical Activity Questionnaire-Short Form; PCS, Perceived Competence Scales; TSRQ, Treatment SelfRegulation Questionnaire.

this context to greater affect clinicians' and staff's health behaviors and attitudes and to sustain improvements.

There are a number of descriptive studies about clinicians' health behavior, yet there are sparse published studies that address onsite interventions for promoting healthy behaviors in this context. ${ }^{7-9}$ Ben-Arye et $\mathrm{al}^{4}$ found that an intense intervention for clinicians and office staff can promote lifestyle self-awareness. Increased self-awareness helped clinicians and office staff make positive attitude changes regarding lifestyle changes. Crawford et $\mathrm{al}^{28}$ conducted a randomized study in small group of Women, Infants, and Children clinics and found that a wellness program improved counselors' personal habits and health beliefs in the intervention group. Furthermore, more intervention site staff reported that their health behaviors set an example for WIC participants. ${ }^{28}$ The current study's strengths include the sample size and diversity of participants, length of follow-up of participants (14 months), randomization of sites to reduce selection bias, and use of validated measures. Future articles will examine the effects of other components of the AIM-HI intervention on selected practice, personnel, and patient outcomes.

\section{Conclusions}

Participation in a practice-wide effort to implement the AIM-HI approach did not significantly improve health behaviors of clinicians and office staff. Staff and clinicians showed short-term improvements in attitudes regarding improving dietary behaviors. The practices involved in this study were already highly motivated and interested in health behavior change, so change might have been hard to detect. This study indicates that, like the patients they care for, clinicians and office staff are resistant to health behavior change. The impact this lack of personal change may have on carrying the message of lifestyle behavioral change to patients requires further study.

The authors acknowledge the practice clinicians and staff who participated in the AIM-HI research project.

\section{References}

1. American Academy of Family Physicians. Americans in Motion-Healthy Interventions (AIM-HI). Available from: http://www.aafp.org/online/en/home/ clinical/publichealth/aim.html. Accessed 25 October 2010. 
2. Whitlock EP, Orleans CT, Pender N, Allan J. Evaluating primary care behavioral counseling interventions: An evidence-based approach. Am J Prev Med 2002;22:267-84.

3. U.S. Department of Health and Human Services. Healthy People 2020. Available from: http://www. healthypeople.gov/2020/default.aspx. Accessed 28 July 2011.

4. Ben-Arye E, Lear A, Hermoni D, Margalit RS. Promoting lifestyle self-awareness among the medical team by the use of an integrated teaching approach: a primary care experience. J Altern Complement Med 2007;13:461-9.

5. Novack DH, Suchman AL, Clark W, Epstein RM, Najberg E, Kaplan C. Calibrating the physician. Personal awareness and effective patient care. Working Group on Promoting Physician Personal Awareness, American Academy on Physician and Patient. JAMA 1997;278:502-9.

6. Oberg EB, Frank E. Physicians' health practices strongly influence patient health practices. J R Coll Physicians Edinb 2009;39:290-1.

7. Bazargan M, Makar M, Bazargan-Hejazi S, Ani C, Wolf KE. Preventive, lifestyle, and personal health behaviors among physicians. Acad Psychiatry 2009; 33:289-95.

8. McGrady FP, McGlade KJ, Cupples ME, Tully MA, Hart N, Steele K. Questionnaire survey of PHysical activITy in General Practitioners (PHIT GP Study). Ulster Med J 2007;76:91-7.

9. Quintela J, Main DS, Pace WD, Staton EW, Black K. LEAP-a brief intervention to improve activity and diet: a report from CaReNet and HPRN. Ann Fam Med 2005;3(Suppl 2):S52-4.

10. Frank E, Breyan J, Elon L. Physician disclosure of healthy personal behaviors improves credibility and ability to motivate. Arch Fam Med 2000;9:287-90.

11. Glasgow RE, Emont S, Miller DC. Assessing delivery of the five 'As' for patient-centered counseling. Health Promot Int 2006;21:245-55.

12. Prochaska JO, DiClemente CC, Norcross JC. In search of how people change. Applications to addictive behaviors. Am Psychol 1992;47:1102-14.

13. Craig CL, Marshall AL, Sjöström M, et al. International physical activity questionnaire: 12-country reliability and validity. Med Sci Sports Exerc 2003;35: 1381-95.

14. Rifas-Shiman SL, Willett WC, Lobb R, Kotch J, Dart C, Gillman MW. PrimeScreen, a brief dietary screening tool: reproducibility and comparability with both a longer food frequency questionnaire and biomarkers. Public Health Nutr 2001;4:249-54.

15. Prochaska JO. Cancer Prevention Research Center Measures. Exercise: stages of change-short form.
Available from: http://www.uri.edu/research/cprc/ Measures/Exercise02.htm. Accessed August 18, 2011.

16. Breuer GS, Orbach H, Elkayam O, et al. Use of complementary and alternative medicine among patients attending rheumatology clinics in Israel. Isr Med Assoc J 2006;8:184-7.

17. Williams GC, Grow VM, Freedman ZR, Ryan RM, Deci EL. Motivational predictors of weight loss and weight-loss maintenance. J Pers Soc Psychol 1996; 70:115-26.

18. Williams GC, Rodin GC, Ryan RM, Grolnick WS, Deci EL. Autonomous regulation and long-term medication adherence in adult outpatients. Health Psychol 1998;17:269-76.

19. Williams, G.C., Z.R. Freedman, and E.L. Deci,. Supporting autonomy to motivate patients with diabetes for glucose control. Diabetes Care 21(10): p. 1644-51, 1998.

20. Williams GC, Deci EL. Internalization of biopsychosocial values by medical students: a test of selfdetermination theory. J Pers Soc Psychol 1996;70: 767-79.

21. Marcus BH, Selby VC, Niaura RS, Rossi JS. Selfefficacy and the stages of exercise behavior change. Req Q Exerc Sport 19921;63:60-6.

22. Boxwala AA, Dierks M, Keenan M, et al. Organization and representation of patient safety data: current status and issues around generalizability and scalability. J Am Med Inform Assoc 2004;11:468-78.

23. Williams GC, Minicucci DS, Kouides RW, et al. Self-determination, smoking, diet and health. Health Educ Res 2002;17:512-21.

24. ter Bogt NC, Bemelmans WJ, Beltman FW, Broer J, Smit AJ, van der Meer K. Preventing weight gain: one-year results of a randomized lifestyle intervention.. Am J Prev Med 2009;37:270-7.

25. Carroll S, Borkoles E, Polman R. Short-term effects of a non-dieting lifestyle intervention program on weight management, fitness, metabolic risk, and psychological well-being in obese premenopausal females with the metabolic syndrome. Appl Physiol Nutr Metab 2007;32:125-42.

26. Diehl HA. Coronary risk reduction through intensive community-based lifestyle intervention: the Coronary Health Improvement Project (CHIP) experience. Am J Cardiol 1998;82(10B):83T-7T.

27. Perri MG, Limacher MC, Durning PE, et al. Extended-care programs for weight management in rural communities: the treatment of obesity in underserved rural settings (TOURS) randomized trial. Arch Intern Med 2008;168:2347-54.

28. Crawford PB, Gosliner W, Strode P, et al. Walking the talk: Fit WIC wellness programs improve selfefficacy in pediatric obesity prevention counseling. Am J Public Health 2004;94:1480-5. 